\title{
Codomain Scale Space and Regularization for High Angular Resolution Diffusion Imaging
}

\author{
Luc Florack \\ Department of Mathematics and Computer Science \& Department of Biomedical Engineering \\ Eindhoven University of Technology, PO Box 513, 5600 MB Eindhoven, The Netherlands \\ L.M.J.Floracketue.nl
}

\begin{abstract}
Regularization is an important aspect to be reckoned with in high angular resolution diffusion imaging (HARDI), since, unlike with DTI, there is no a priori regularity of raw data in the co-domain, i.e. considered as a multispectral signal for fixed spatial position. HARDI preprocessing is therefore a crucial step prior to any subsequent analysis, and some insight in regularization paradigms and their interrelations is compulsory.

In this paper we posit a codomain scale space regularization paradigm that has hitherto not been applied in the context of HARDI. Unlike previous (first and second order) schemes it is based on infinite order regularization, yet can be fully operationalized. We furthermore establish a closedform relation with first order Tikhonov regularization via the Laplace transform.
\end{abstract}

Keywords: high angular resolution diffusion imaging (HARDI); diffusion tensor imaging (DTI); regularization; scale space

\section{Introduction}

By virtue of the directional dependence of water diffusion characteristics, fibrous tissue such as white matter and muscle can be studied in vivo and non-invasively with the help of MRI diffusion imaging protocols and corresponding physical/mathematical models. These are invariably based on variants of high angular resolution diffusion imaging (HARDI), such as Tuch's orientation distribution function (ODF) [26], the higher order diffusion tensor model and the diffusion orientation transform (DOT) by Özarslan et al. [21, 22], and the diffusion tensor distribution model by Jian et al. [13].
The desire to account for signal attenuation and diffusivity profiles more complex than can be realistically coped with by the conventional rank-2 DTI model-introduced by Basser et al. [2, 3], cf. also Le Bihan et al. [17], and itself a HARDI regularization model by construction-has led to a concomitant need for regularization techniques, such as proposed by Descoteaux et al. [4, 5] and by Hess et al. [12], since there is no a priori smoothness of acquisition data.

In this conceptual paper we posit a new regularization scheme based on an extension of classical scale space theory, and show that it generalizes Tikhonov regularization by establishing a well-posed, analytically closed-form procedure for mapping the scale space regularized signal to the Tikhonov regularized signal via the Laplace transform. The inverse mapping exists (thus the schemes are formally equivalent), but is ill-posed and thus operationally void. Although perhaps a minor contribution for the practically minded, the paper does offer new insight in the relationship between regularization schemes of various orders. Insight provided by our new analytical result and its connection to established results may be decisive in one's choice for HARDI preprocessing. It is clear that, in any case, the choice of regularization will affect the outcome of subsequent analysis (cf. the comparison study by Descoteaux et al., loc. cit.), thus insight is of fundamental importance per se.

\section{Theory}

\subsection{Notation}

Let $S: \Omega \rightarrow \mathbb{R}$ denote a given raw HARDI signal confined to the unit sphere $\Omega:\|x\|=1, x \in \mathbb{R}^{3}$. $\Omega$ may be parameterized using two coordinates, $\xi^{\mu}, \mu=1,2$, say. An obvious choice would be the polar angles $\xi=\left(\xi^{1}, \xi^{2}\right)=$ $(\theta, \phi) \in[0, \pi] \times[0,2 \pi)$, given in terms of $3 \mathrm{D}$ Cartesian 
coordinates $^{1} x^{i}, i=1,2,3$, by

$$
\left\{\begin{array}{l}
x^{1}=\sin \theta \cos \phi \\
x^{2}=\sin \theta \sin \phi \\
x^{3}=\cos \theta
\end{array}\right.
$$

The components of the Riemannian metric for the unit sphere embedded in Euclidean 3 -space $\mathbb{R}^{3}$ are given by

$$
g_{\mu \nu}=\frac{\partial x^{i}}{\partial \xi^{\mu}} \eta_{i j} \frac{\partial x^{j}}{\partial \xi^{\nu}}
$$

in which $\eta_{i j}$ denote the components of the Euclidean metric of the embedding space. Using polar and Cartesian coordinates for $\Omega$ and $\mathbb{R}^{3}$, respectively, we obtain the familiar matrix representations

$$
\boldsymbol{g}=\left(\begin{array}{cc}
1 & 0 \\
0 & \sin ^{2} \theta
\end{array}\right) \quad \text { resp. } \boldsymbol{\eta}=\left(\begin{array}{lll}
1 & 0 & 0 \\
0 & 1 & 0 \\
0 & 0 & 1
\end{array}\right)
$$

In particular, $\sqrt{g} d \xi=\sqrt{\operatorname{det} \boldsymbol{g}} d \xi^{1} d \xi^{2}=\sin \theta d \theta d \phi$ is the invariant volume element.

However, for the sake of transparency and generality, as well as to facilitate mathematical manipulations below, we will initially allow for general, closed (smooth, not necessarily embedded) manifolds and coordinate conventions, and only later on specify results for the unit sphere endowed with the conventional spherical coordinates, Eqs. (1-3).

With $D_{\mu}$ we denote the covariant derivative with respect to $x^{\mu}$ induced by the metric $g_{\mu \nu}$. Recall that by construction the metric is "covariantly constant": $D_{\rho} g_{\mu \nu}=0$, whence also $D_{\mu} g=0$. One can take this as the definition of the covariant derivative [24].

We will assume that $\Omega$ is a single "fibre" over the Euclidean space $\mathbb{R}^{3}$ at some implicit base point $x \in \mathbb{R}^{3}$. More specifically, in the context of HARDI, $\Omega$ is the domain of definition of the diffusive attenuation signal $S(\xi)$ or the diffusion coefficient $D(\xi)$ defined via the general Stejskal-Tanner formula $[21,25]$

$$
S(\xi)=S_{0} \exp (-b D(\xi)) .
$$

(That is, we do not make the DTI assumption that $D(\xi)$ can be expressed as rank-two tensor. The functions $S(\xi)$ and $D(\xi)$ are thus essentially arbitrary, and Eq. (4) holds by definition.)

In what follows we shall consider regularization of the function $S(\xi)$, but the method proposed can be equally well applied to any other $L^{2}(\Omega)$-function on $\Omega$, notably to $D(\xi)$, or to such derived quantities as the DOT [22].

\footnotetext{
${ }^{1}$ We will adhere to the convention that Latin indices label coordinates of the embedding space $\mathbb{R}^{3}$, while Greek indices label parameters of the embedded submanifold.
}

\subsection{Regularization}

Consider the following functionals ${ }^{2}$, in which $S_{t}: \Omega \rightarrow \mathbb{R}$ (referred to henceforth as the scale space extension of the raw signal $S$ ) and $\widetilde{S}_{s}: \Omega \rightarrow \mathbb{R}$ (the first order Tikhonov regularization of $S$ ) are two alternative regularizations of the raw HARDI signal $S: \Omega \rightarrow \mathbb{R}$, viz.

$$
\begin{aligned}
& E\left(S_{t}\right)=\int_{\Omega}\left(S(\xi)-S_{t}(\xi)\right)^{2} D \xi \\
& \quad+\sum_{k \geq 1} \frac{t^{k}}{k !} \int_{\Omega} D_{\mu_{1}} \ldots D_{\mu_{k}} S_{t}(\xi) D^{\mu_{1}} \ldots D^{\mu_{k}} S_{t}(\xi) D \xi
\end{aligned}
$$

in which $D^{\mu}$ is shorthand for $g^{\mu \nu}(\xi) D_{\nu}$, and in which $D \xi=\sqrt{g(\xi)} d \xi$, with $d \xi=d \xi^{1} d \xi^{2}$, denotes the invariant measure on $\Omega$. Furthermore

$$
\begin{array}{r}
\widetilde{E}\left(\widetilde{S}_{s}\right)=\int_{\Omega}\left(S(\xi)-\widetilde{S}_{s}(\xi)\right)^{2} D \xi \\
+\quad s \int_{\Omega} D_{\mu} \widetilde{S}_{s}(\xi) D^{\mu} \widetilde{S}_{s}(\xi) D \xi
\end{array}
$$

Equivalently, one may write (notice the minus signs)

$$
\begin{aligned}
& E\left(S_{t}\right)=\int_{\Omega}\left(S(\xi)-S_{t}(\xi)\right)^{2} D \xi \\
& +\sum_{k \geq 1} \frac{(-t)^{k}}{k !} \int_{\Omega} S_{t}(\xi) \Delta_{g}^{k} S_{t}(\xi) D \xi
\end{aligned}
$$

respectively

$$
\begin{gathered}
\widetilde{E}\left(\widetilde{S}_{s}\right)=\int_{\Omega}\left(S(\xi)-\widetilde{S}_{s}(\xi)\right)^{2} D \xi \\
-s \int_{\Omega} \widetilde{S}_{s}(\xi) \Delta_{g} \widetilde{S}_{s}(\xi) D \xi
\end{gathered}
$$

in which $\Delta_{g}=g^{\mu \nu}(\xi) D_{\mu} D_{\nu}$ denotes the self-adjoint Laplace-Beltrami operator on $\Omega$, i.e. if $f: \Omega \rightarrow \mathbb{R}$ is a scalar function on $\Omega$, then we have

$$
\Delta_{g} f=\frac{1}{\sqrt{g}} \partial_{\mu}\left(g^{\mu \nu} \sqrt{g} \partial_{\nu} f\right) .
$$

These rewritings are possible by virtue of the fact that (i) the product rule for differentiation holds for covariant derivatives, enabling partial integration in formally the same way as applicable to ordinary derivatives, and (ii) the boundary $\partial \Omega$ of $\Omega$ is empty. In particular, in terms of polar coordinates for the unit sphere, Eqs. (1-3):

$$
\Delta_{g} f(\theta, \phi)=\left(\frac{1}{\sin ^{2} \theta} \frac{\partial^{2}}{\partial \phi^{2}}+\frac{1}{\sin \theta} \frac{\partial}{\partial \theta}\left(\sin \theta \frac{\partial}{\partial \theta}\right)\right) f(\theta, \phi) .
$$

\footnotetext{
${ }^{2}$ Index summation applies to pairs of identical upper and lower indices.
} 
Using covariant constancy of the metric, and the fact that $\Delta_{g}^{k}$ is self-adjoint for any $k=\mathbb{Z}_{0}^{+}$, it is straightforward to derive the corresponding Euler-Lagrange equations. For Eq. (5) this yields the "infinite order" PDE

$$
S(\xi)=\sum_{k \geq 0} \frac{(-t)^{k}}{k !} \Delta_{g}^{k} S_{t}(\xi)=\exp \left(-t \Delta_{g}\right) S_{t}(\xi) .
$$

Inversion yields the solution

$$
S_{t}(\xi)=\sum_{k \geq 0} \frac{t^{k}}{k !} \Delta_{g}^{k} S(\xi)=\exp \left(t \Delta_{g}\right) S(\xi) .
$$

Notice that it satisfies the heat equation on the sphere. This is analogous to the standard scale space representation in the Euclidean plane $[6,8,10,11,14,15,16,18,20,23$, 27], with the Euclidean Laplace operator $\Delta$ on $\mathbb{R}^{n}$ formally replaced by $\Delta_{g}$. The (dimensionless) parameter ${ }^{3} t \in \mathbb{R}^{+}$ controls the (inverse) angular resolution.

The Euler-Lagrange equation corresponding to the Tikhonov regularized functional, Eq. (6), is given by the second order elliptic PDE

$$
S(\xi)=\left(I-s \Delta_{g}\right) \widetilde{S}_{s}(\xi)
$$

Its solution is the well-known Tikhonov-regularized signal

$$
\widetilde{S}_{s}(\xi)=\left(I-s \Delta_{g}\right)^{-1} S(\xi) .
$$

Henceforth we specify results for the spherical geometry appropriate for HARDI representations, recall Eqs. (1-4).

Expressed in terms of spherical coordinates it is natural to decompose the functions on left and right hand side of Eqs. (12) and (14) relative to the orthonormal basis of spherical harmonics, cf. Frank [9] and Alexander et al. [1], since this diagonalizes the Laplace-Beltrami operator:

$$
\Delta_{g} Y_{\ell}^{m}=-\ell(\ell+1) Y_{\ell}^{m}
$$

for any $\ell \in \mathbb{Z}_{0}^{+}$and $m \in\{-\ell,-\ell+1, \ldots, \ell-1, \ell\}$. We employ the following convention for spherical harmonics, in which $P_{\ell}^{m}$ are the associated Legendre polynomials ${ }^{4}$ :

$$
\begin{gathered}
Y_{\ell}^{m}(\cos \theta)=\sqrt{\frac{(2 \ell+1)(\ell-m) !}{4 \pi(\ell+m) !}} e^{i m \phi} P_{\ell}^{m}(\cos \theta) . \\
P_{\ell}^{m}(z)=\frac{(-1)^{m}}{2^{\ell} \ell !}\left(1-z^{2}\right)^{\frac{m}{2}} \frac{d^{\ell+m}}{d z^{\ell+m}}\left(z^{2}-1\right)^{\ell},
\end{gathered}
$$

\footnotetext{
${ }^{3}$ The operator $\exp \left(t \Delta_{g}\right)$ is bounded and defines a strongly continuous semigroup for $t \in \mathbb{R}^{+} \cup\{0\}$.

${ }^{4} \mathrm{Cf}$. functions.wolfram.com for further properties of $Y_{\ell}^{m}$ and $P_{\ell}^{m}$.
}

with $-1 \leq z \leq 1$. Setting

$$
S(\theta, \phi)=\sum_{\ell, m}^{*} c_{\ell m}(0) Y_{\ell}^{m}(\theta, \phi),
$$

in which the asterisk indicates summation over the effective indices $\ell \in \mathbb{Z}_{0}^{+}, m \in\{-\ell,-\ell+1, \ldots, \ell-1, \ell\}$, yields

$$
S_{t}(\theta, \phi)=\sum_{\ell, m}^{*} c_{\ell m}(t) Y_{\ell}^{m}(\theta, \phi),
$$

with

$$
c_{\ell m}(t)=e^{-t \ell(\ell+1)} c_{\ell m}(0) .
$$

Notice the analogy with the $e^{-t\|\omega\|^{2}}$-attenuation of high frequency components of scalar images in the Euclidean plane under Gaussian blurring.

Recall that the coefficients in Eq. (18) are determined as follows:

$$
c_{\ell m}(0)=\int_{0}^{2 \pi} \int_{0}^{\pi} S(\theta, \phi) Y_{\ell}^{-m}(\theta, \phi) \sin \theta d \theta d \phi,
$$

by virtue of $Y_{\ell}^{-m}=\left(Y_{\ell}^{m}\right)^{*}$ and orthonormality:

$$
\int_{0}^{2 \pi} \int_{0}^{\pi} Y_{\ell}^{m}(\theta, \phi)^{*} Y_{\ell^{\prime}}^{m^{\prime}}(\theta, \phi) \sin \theta d \theta d \phi=\delta_{m m^{\prime}} \delta_{\ell \ell^{\prime}}
$$

Eqs. (19-21) summarize the operational scheme for regularizing the HARDI signal according to the "infinite order" regularization scheme defined implicitly via minimization of Eq. (5).

Similarly, using Eq. (18) together with Eqs. (14-15), we have for the first order regularization scheme:

$$
\widetilde{S}_{s}(\theta, \phi)=\sum_{\ell, m}^{*} \widetilde{c}_{\ell m}(s) Y_{\ell}^{m}(\theta, \phi)
$$

with

$$
\widetilde{c}_{\ell m}(s)=\frac{c_{\ell m}(0)}{1+s \ell(\ell+1)},
$$

cf. Eq. (20). This is the analogy of the $1 /\left(1+s\|\omega\|^{2}\right)$ attenuation of high frequency components of scalar images in the Euclidean plane after Tikhonov regularization.

The asymptotic cases are as expected:

$$
\begin{gathered}
\lim _{t \rightarrow 0^{+}} S_{t}(\theta, \phi)=\lim _{s \rightarrow 0^{+}} \widetilde{S}_{s}(\theta, \phi)=S(\theta, \phi), \\
\lim _{t \rightarrow \infty} S_{t}(\theta, \phi)=\lim _{s \rightarrow \infty} \widetilde{S}_{s}(\theta, \phi)= \\
\frac{1}{4 \pi} \int_{0}^{2 \pi} \int_{0}^{\pi} S(\theta, \phi) \sin \theta d \theta d \phi
\end{gathered}
$$


i.e., in the hypothetical limit of infinite angular resolution Eqs. (19-21) and (23-24) reproduce the raw signal, whereas the limit of vanishing angular resolution corresponds to a complete averaging of this raw signal over the sphere. Indeed, the signals $\widetilde{S}_{s}$ and $S_{t}$ are qualitatively similar, and are both viable regularizations of the raw signal on the unit sphere. Which of these two alternatives is to be preferred, if any, probably depends on the application context.

Of course one may want to opt for regularizing the apparent diffusion coefficient $D(\xi)$ instead of the diffusive attenuation signal $S(\xi)$, or any other suitably defined HARDIrelated distribution on the sphere, such as mentioned in the introduction, by exactly the same procedures. For instance, $\mathrm{H}_{2} \mathrm{O}$-displacement probability distributions derived from the observed MR signal attenuation, such as captured by the DOT [22], typically exhibit profiles that more closely reflect actual fiber orientations, as opposed to the MR attenuation signal or related diffusivity profiles themselves.

\subsection{Tikhonov versus Scale Space Regularization}

Following a similar approach as done previously in the context of regularization in the Euclidean plane [7], our aim is to establish an exact connection between the two alternative regularizations, Eqs. (12) and (14). (An approximate relationship is immediately apparent from the fact that the resolvent operator in the latter equation can be seen as a first order approximation of the heat operator in the former, cf. Nielsen et al. in the context of Euclidean images [19].)

The explicit relation between the two representations, Eqs. (12) and (14), is as follows:

$$
\begin{aligned}
\widetilde{S}_{s}(\xi) & \stackrel{\text { def }}{=} \frac{1}{s} \int_{0}^{\infty} \exp \left(-\frac{t}{s}\right) S_{t}(\xi) d t \\
= & \frac{1}{s} \int_{0}^{\infty} \exp \left(-\frac{t}{s}\right) \exp \left(t \Delta_{g}\right) S(\xi) d t \\
= & \left(I-s \Delta_{g}\right)^{-1} S(\xi)
\end{aligned}
$$

Alternatively, we arrive at this same result by noticing that

$$
\tilde{c}_{\ell m}(s)=\frac{1}{s} \int_{0}^{\infty} \exp \left(-\frac{t}{s}\right) c_{\ell m}(t) d t,
$$

which at the same time provides an operationally welldefined scheme for the operator identity Eq. (27), recall Eqs. (20) and (24).

Notice the formal relationship, analogous to Eqs. (27) and (28), between the physical resolution parameters:

$$
\frac{1}{s} \int_{0}^{\infty} \exp \left(-\frac{t}{s}\right) t d t=s
$$

Thus we may formally interpret the Tikhonov parameter $s$ as the expectation value of the scale space parameter $t$ under a negative-exponential scale distribution. The Tikhonovregularized signal $\widetilde{S}_{s}$ or, equivalently, the set of coefficients $\widetilde{c}_{\ell m}(s)$, apparently arises via a similar scale superposition from the scale space signal $S_{t}$, respectively the set $c_{\ell m}(t)$.

Eq. (27) shows that the functions $S_{t}$ and $\widetilde{S}_{s}$ basically form a Laplace transform pair. Thus formally speaking the two schemes are equivalent. However, it is well-known that the inverse operation is ill-posed, thus operationally void. Thus we may state that scale space regularization in the sense of the proposed "infinite order" scheme is a true generalization of first order Tikhonov regularization, viz. to the extent that the latter can always be derived a posteriori (for any fixed Tikhonov regularization parameter $s \in \mathbb{R}^{+}$) given the former (as a function of the scale parameter $t \in \mathbb{R}^{+}$), but-in practice-not vice versa. Cf. the literature for details in the Euclidean plane [7].

\subsection{Other Regularization Schemes}

Descoteaux et al. propose another regularization scheme, one in which the regularization term is a quadratic functional of second order derivatives [4, 5]. By virtue of spherical harmonic decomposition also this scheme admits a closed-form solution. An exact relation of the type discussed in the previous section with either first order Tikhonov or infinite order scale space regularization is, however, hitherto unknown. Again, also this second order scheme is qualitatively similar to the ones proposed here. Based on an empirical comparison study the authors express a slight preference for their second order scheme over the first order one, but this conclusion is probably context and application dependent. In any case, if this preference is somehow related to the order of the scheme, this would itself provide additional justification - and is indeed what motivated us in the first place - to study regularization to any order. The availability of analytical closed-form expressions is an advantage that all these schemes have in common. For many practical purposes the details of the regularization scheme employed may be less decisive than the way in which the scheme is used.

In principle, Eq. (5) is easily adapted so as to account for arbitrary (quadratic) regularization schemes by replacing the specific choice of coefficients ${ }^{5}$ (i.e. $t^{k}$ ) by any suitably chosen sequence $\lambda_{k}$ (leading to convergence). But (to the best

\footnotetext{
${ }^{5}$ With only one parameter at hand, physical scaling arguments naturally single out the monomial $t^{k}$-behaviour of the $k$-th order coefficients in Eq. (5). The factorial $k$ ! is a natural dimensionless weight as it counts the number of terms in the $k$-th order contraction.
} 
of our knowledge) closed-form solutions are not easily obtained for cases other than the ones discussed here.

Finally, yet another form of regularization is achieved by "brute force" truncation of the series Eq. (18) at some $\ell=$ $\ell_{\max }$, the analogue of ideal low-pass filtering. For this case there exists no simple functional minimization formulation.

\section{Conclusion}

We have established a linear filtering paradigm for HARDI codomain regularization on the unit sphere, based on energy minimization with Tikhonov regularization terms up to infinite order, in analytically closed form. We have shown that it basically generalizes scale space theory from the Euclidean plane to non-Euclidean Riemannian manifolds, and elaborated on the specific, spherical geometry appropriate for various HARDI based representations proposed in the literature. Subsequently we have derived an explicit mapping that relates this scale space representation to first order Tikhonov regularization via the Laplace transform. Expressed in terms of coefficients relative to the basis of spherical harmonics the corresponding one-parameter smoothing filter is as straightforwardly implemented as any of the existing (first and second order) Tikhonov-like schemes described in the literature.

Qualitatively, these schemes are similar in their behaviour as a function of the free parameter that couples the differential regularization terms to the data term, and identical in their asymptotics. Whether the new representation has any advantages over existing ones remains therefore to be seen. One could undertake a similar detailed endeavour for mutual comparison as pursued by Descoteaux et al. [4, 5]. However, it is not unlikely that the outcome of such a comparison experiment depends on the target criterion (in the above case detection of crossing fibers and reduction of angular error), and on a combination of various intrinsic factors, such as the spherical harmonic order taken into account and the selection of the free regularization parameter. One should note in this respect that it is neither trivial to determine optimal factors within each scheme, nor to compare them across schemes.

We believe that no decisive generic conclusion can be drawn (as yet). In any case, basic insight in the various regularization schemes and their interrelations remains of utmost importance, since such schemes are often necessary and may affect any further HARDI analysis in a crucial way. The main purpose of this conceptual paper has been to contribute to this insight via a generalization of existing regularization schemes.

\section{Acknowledgements.}

The Netherlands Organisation for Scientific Research (NWO) is gratefully acknowledged for financial support.

\section{References}

[1] D. C. Alexander, G. J. Barker, and S. R. Arridge. Detection and modeling of non-Gaussian apparent diffusion coefficient profiles in human brain data. Magnetic Resonance in Medicine, 48(2):331-340, 2002.

[2] P. J. Basser, J. Mattiello, and D. Le Bihan. Estimation of the effective self-diffusion tensor from the NMR spin echo. Journal of Magnetic Resonance, 103:247-254, 1994.

[3] P. J. Basser, J. Mattiello, and D. Le Bihan. MR diffusion tensor spectroscopy and imaging. Biophysics Journal, 66(1):259-267, 1994.

[4] M. Descoteaux, E. Angelino, S. Fitzgibbons, and R. Deriche. Apparent diffusion coefficients from high angular resolution diffusion imaging: Estimation and applications. Magnetic Resonance in Medicine, 56:395-410, 2006.

[5] M. Descoteaux, E. Angelino, S. Fitzgibbons, and R. Deriche. Regularized, fast, and robust analytical Q-ball imaging. Magnetic Resonance in Medicine, 58:497-510, 2007.

[6] R. Duits, L. Florack, J. de Graaf, and B. ter Haar Romeny. On the axioms of scale space theory. Journal of Mathematical Imaging and Vision, 20(3):267-298, 2004.

[7] L. Florack, R. Duits, and J. Bierkens. Tikhonov regularization versus scale space: A new result. In Proceedings of the 11th International Conference on Image Processing (Singapore, October 24-27, 2004), pages 271-274. IEEE, 2004.

[8] L. M. J. Florack. Image Structure, volume 10 of Computational Imaging and Vision Series. Kluwer Academic Publishers, Dordrecht, The Netherlands, 1997.

[9] L. R. Frank. Characterization of anisotropy in high angular resolution diffusion-weighted MRI. Magnetic Resonance in Medicine, 47(6):1083-1099, 2002.

[10] L. D. Griffin and M. Lillholm, editors. Scale-Space Methods in Computer Vision: Proceedings of the Fourth International Conference, Scale-Space 2003, Isle of Skye, UK, volume 2695 of Lecture Notes in Computer Science. SpringerVerlag, Berlin, June 2003.

[11] B. M. t. Haar Romeny, L. M. J. Florack, J. J. Koenderink, and M. A. Viergever, editors. Scale-Space Theory in Computer Vision: Proceedings of the First International Conference, Scale-Space'97, Utrecht, The Netherlands, volume 1252 of Lecture Notes in Computer Science. Springer-Verlag, Berlin, July 1997.

[12] C. P. Hess, P. Mukherjee, E. T. Tan, D. Xu, and D. B. Vigneron. Q-ball reconstruction of multimodal fiber orientations using the spherical harmonic basis. Magnetic Resonance in Medicine, 56:104-117, 2006.

[13] B. Jian, B. C. Vemuri, E. Özarslan, P. R. Carney, and T. H. Mareci. A novel tensor distribution model for the diffusionweighted MR signal. NeuroImage, 37:164-176, 2007. 
[14] M. Kerckhove, editor. Scale-Space and Morphology in Computer Vision: Proceedings of the Third International Conference, Scale-Space 2001, Vancouver, Canada, volume 2106 of Lecture Notes in Computer Science. Springer-Verlag, Berlin, July 2001.

[15] R. Kimmel, N. Sochen, and J. Weickert, editors. Scale Space and PDE Methods in Computer Vision: Proceedings of the Fifth International Conference, Scale-Space 2005, Hofgeismar, Germany, volume 3459 of Lecture Notes in Computer Science. Springer-Verlag, Berlin, April 2005.

[16] J. J. Koenderink. The structure of images. Biological Cybernetics, 50:363-370, 1984.

[17] D. Le Bihan, J.-F. Mangin, C. Poupon, C. A. Clark, S. Pappata, N. Molko, and H. Chabriat. Diffusion tensor imaging: Concepts and applications. Journal of Magnetic Resonance Imaging, 13:534-546, 2001.

[18] T. Lindeberg. Scale-Space Theory in Computer Vision. The Kluwer International Series in Engineering and Computer Science. Kluwer Academic Publishers, Dordrecht, The Netherlands, 1994.

[19] M. Nielsen, L. M. J. Florack, and R. Deriche. Regularization, scale-space and edge detection filters. Journal of Mathematical Imaging and Vision, 7(4):291-307, October 1997.

[20] M. Nielsen, P. Johansen, O. F. Olsen, and J. Weickert, editors. Scale-Space Theories in Computer Vision: Proceedings of the Second International Conference, Scale-Space'99, Corfu, Greece, volume 1682 of Lecture Notes in Computer Science. Springer-Verlag, Berlin, September 1999.

[21] E. Özarslan and T. H. Mareci. Generalized diffusion tensor imaging and analytical relationships between diffusion tensor imaging and high angular resolution imaging. Magnetic Resonance in Medicine, 50:955-965, 2003.

[22] E. Özarslan, T. M. Shepherd, B. C. Vemuri, S. J. Blackband, and T. H. Mareci. Resolution of complex tissue microarchitecture using the diffusion orientation transform (DOT). NeuroImage, 31:1086-1103, 2006.

[23] F. Sgallari, A. Murli, and N. Paragios, editors. Scale Space and Variational Methods in Computer Vision: Proceedings of the First International Conference, SSVM 2007, Ischia, Italy, volume 4485 of Lecture Notes in Computer Science. Springer-Verlag, Berlin, May-June 2007.

[24] M. Spivak. Differential Geometry, volume 1-5. Publish or Perish, Berkeley, 1975.

[25] E. O. Stejskal and J. E. Tanner. Spin diffusion measurements: Spin echoes in the presence of a time-dependent field gradient. Journal of Computational Physics, 42:288-292, 1965.

[26] D. S. Tuch. Q-ball imaging. Magnetic Resonance in Medicine, 52:1358-1372, 2004.

[27] A. P. Witkin. Scale-space filtering. In Proceedings of the International Joint Conference on Artificial Intelligence, pages 1019-1022, Karlsruhe, Germany, 1983. 